\title{
Is No-Tension Design of Concrete or Rock Structures Always SAFE? - Fracture ANAlysis
}

\author{
By Zdeněk P. Bažant, ${ }^{1}$ Fellow, ASCE
}

\begin{abstract}
Plain concrete structures such as dams or retaining walls, as well as rock structures such as tunnels, caverns, excavations, and rock slopes, have commonly been designed by elastic-perfectly plastic analysis in which the tensile yield strength of the material is taken as zero. The paper analyzes the safety of this "notension" design in the light of the finiteness of the tensile strength of concrete or the tensile strength of rock between the joints. Through examples, it is demonstrated that: (1) the calculated length of cracks or cracking zones can correspond to an unstable state; (2) the uncracked ligament of the cross section, available for resisting horizontal shear loads, can be predicted much too large, compared to the fracture mechanics prediction; (3) the calculated load-deflection diagram can lie lower than that obtained by fracture mechanics; (4) the no-tension load capacity for a combination of crack face pressure and loads remote from the crack front, calculated by elastic analysis on the basis of allowable compressive stress, can be higher than that obtained by fracture mechanics; and (5) an increase in the tensile strength of the material can cause the load capacity of the structure to decrease. Due to the size effect, these facts are true not only for zero fracture toughness (no-toughness design) but also for finite fracture toughness provided that the structure size is large enough. Several previous studies on the safety of no-tension design, including the finite-element analysis of a gravity dam, are also reviewed. It is concluded that if the no-tension limit design is used, the safety factors of concrete or rock structures cannot be guaranteed to have the specified values. Fracture mechanics is required for that.
\end{abstract}

\section{INTRODUCTION}

Concrete, and especially dam concrete, is a material of a relatively low and highly variable tensile strength. The same is true in the macroscopic sense of rock masses intersected by a system of joints or preexisting cracks. Like rock joints, construction joints may also have no tensile strength. Therefore, the design of unreinforced concrete structures such as dams or retaining walls, as well as rock structures such as tunnels, caverns, excavations, and rock slopes, has commonly been made under the hypothesis that the material has no tensile strength. Accordingly, in the simplified design of the horizontal cross section of a dam, the distribution of the vertical normal stress has normally been assumed to be triangular within the compressed part of the cross section (WES 1983; BuRec 1987; Jansen 1988).

The "no-tension" design has traditionally been used for masonry - for example stone arches, domes, or pillars. In that case, the hypothesis of no tension is often nearly exactly true, because of negligible strength and the dense spacing of the joints. During the 1960s, the no-tension hypothesis was introduced into finite-element analysis [see, e.g., Zienkiewicz (1971)]. In that case, the no-tension hypothesis is properly implemented as the limit case of plasticity in which the tensile yield strength approaches zero. Various yield criteria can be used, for example, the Rankine criterion or Mohr-Coulomb criterion [see, e.g., Owen and Hinton (1980)]. In the theory of plasticity, it is proven [e.g., Hodge (1959)] that if yield surface $A$ lies within yield surface $B$, the limit load for surface $B$ cannot be lower than the limit load for surface $A$. Thus, for plastic materials, the no-tension design is guaranteed to be on the safe side. Not so, however, for brittle materials.

The failure of brittle materials such as concrete or rock is properly described by fracture mechanics rather than plasticity. Such materials do not fail simultaneously along the entire

${ }^{1}$ Walter P. Murphy Prof. of Civ. Engrg. and Mat. Sci., Northwestern Univ., Evanston, IL 60208.

Note. Associate Editor: Steven L. McCabe. Discussion open until June 1, 1996. To extend the closing date one month, a written request must be filed with the ASCE Manager of Journals. The manuscript for this paper was submitted for review and possible publication on June 6, 1994. This paper is part of the Journal of Structural Engineering, Vol. 122, No. 1, January, 1996. CASCE, ISSN 0733-9445/96/0001-0002-0010/ $\$ 4.00+\$ .50$ per page. Paper No. 8597 failure surface, as required for applicability of the plastic limit analysis. Rather, the failure propagates. Although the initiation of fracture is still governed by tensile strength, dams or tunnels cannot be designed to fail at the initiation of fracture. They must be designed so as to fail only after a large stable crack growth. Instead of tensile strength, the failure is then governed by the condition that the rate at which the potential energy of the structure-load system is released during fracture propagation become equal to the energy per unit crack area that the material can absorb. This is called the fracture energy, $G_{f}$, and represents the most fundamental failure characteristic of the material. In this regard, an important point is that a structure of a higher tensile strength can store more energy and can thus release stored energy during fracture propagation at a higher rate (Bažant 1990). One must, therefore, suspect that an increase of tensile strength could, at least in some cases, reduce the load capacity of the structure (Bažant 1990). In other words, the no-tension design might not always guarantee safety if the material is brittle.

The question of safety of the no-tension design was raised at the dam fracture workshop in Locarno, Switzerland (Dungar et al. 1990), and was intensely debated at the subsequent dam fracture conferences in Boulder, Colorado (Saouma et al. 1991) and Chambéry, France (Bourdarot et al. 1994). By simple examples of analytical solutions of rectangular specimens, it was shown (Bažant 1990) that, according to fracture mechanics, tensile stresses can occur in no-tension plastic designs. Furthermore, the well-known size effect of linear elastic fracture mechanics (Bažant 1984; He et al. 1992) was shown to imply that the no-tension design is not guaranteed to be safe. In a subsequent study (Gioia et al. 1992), a typical gravity dam with a large crack was considered to be overtopped and loaded by gravity, along with water pressure on the upstream face and on the crack faces. Finite-element analysis showed that the load-deflection diagram obtained by fracture mechanics with a finite fracture toughness can lie below (in fact, significantly below) that obtained by no-tension plasticity.

The objective of this paper is twofold: (1) To present several new examples, which are easier to analyze and explain than those presented earlier; (2) to review and interpret the existing finite-element results on the safety of the no-tension design. 
The no-tension hypothesis has, for a long time, been used for reinforced-concrete beams, columns, slabs, and other structures. The reinforced-concrete structures, however, are not of concern here. For them, the safety of the no-tension hypothesis is not disputed, because: (1) The contribution of the tensile capacity of concrete to the load capacity of the structure is small, compared to the contribution of the tensile capacity of steel reinforcement; (2) the reinforcement forces the tensile cracks to be densely distributed, i.e., prevents them from localizing into wide, isolated cracks; and (3) the yielding of steel reinforcement endows the load-deflection diagram of the structure with sufficient ductility, manifested by a prolonged horizontal yield plateau. The yield plateau implies the cause of failure to be a single-degree-of-freedom plastic collapse mechanism rather than localization of damage and propagation of fracture.

\section{EXAMPLE 1: APPLIED FORCE REMOTE FROM CRACK FRONT}

To clarify the potential fallacy of the no-tension design, it is helpful to consider simple specimens for which the solutions according to linear elastic fracture mechanics (LEFM) are available in handbooks such as those by Tada et al. (1985) or Murakami (1987). Let us consider the rectangular concrete specimen in Fig. 1(a). It contains a horizontal crack of length $a=D / 2$ and has a unit thickness, width $D$, and height $H$. It is loaded by the axial force $P$ that has an eccentricity $e$ and is applied at a remote location from the crack. Except for the absence of a shear force, this is similar to the loading of the cross section of a concrete gravity dam. In this first example, let us assume that $e=D / 3$ [Fig. 1(a)].

First consider concrete to be an elastic material with zero tensile strength. Then, the left half of the specimen carries no stresses. This implies that the left half of the specimen is intersected by densely distributed (continuously smeared) cracks, as shown in Fig. 1(b). In the right half, the distribution of the axial compressive stresses is triangular [Fig. 1(b)] and the same in every horizontal cross section because the cross sections remain plane.

In the no-tension design, the basic condition of safety is that a stress distribution with no tensile stresses must exist. If such a distribution is found, any proportional increase of the load will maintain the tension-free state because the material behaves linearly in compression. So, the load capacity is not limited by tensile behavior of the material. Instead, it is limited by compression strength. The compression failure of brittle materials such as concrete or rock consists of propagation of bands of compression splitting cracks and shear damage bands. This failure is also brittle. It does not exhibit a yield plateau. However, analysis of these phenomena is extremely complex.

Therefore, if a stable no-tension state is found, the ultimate
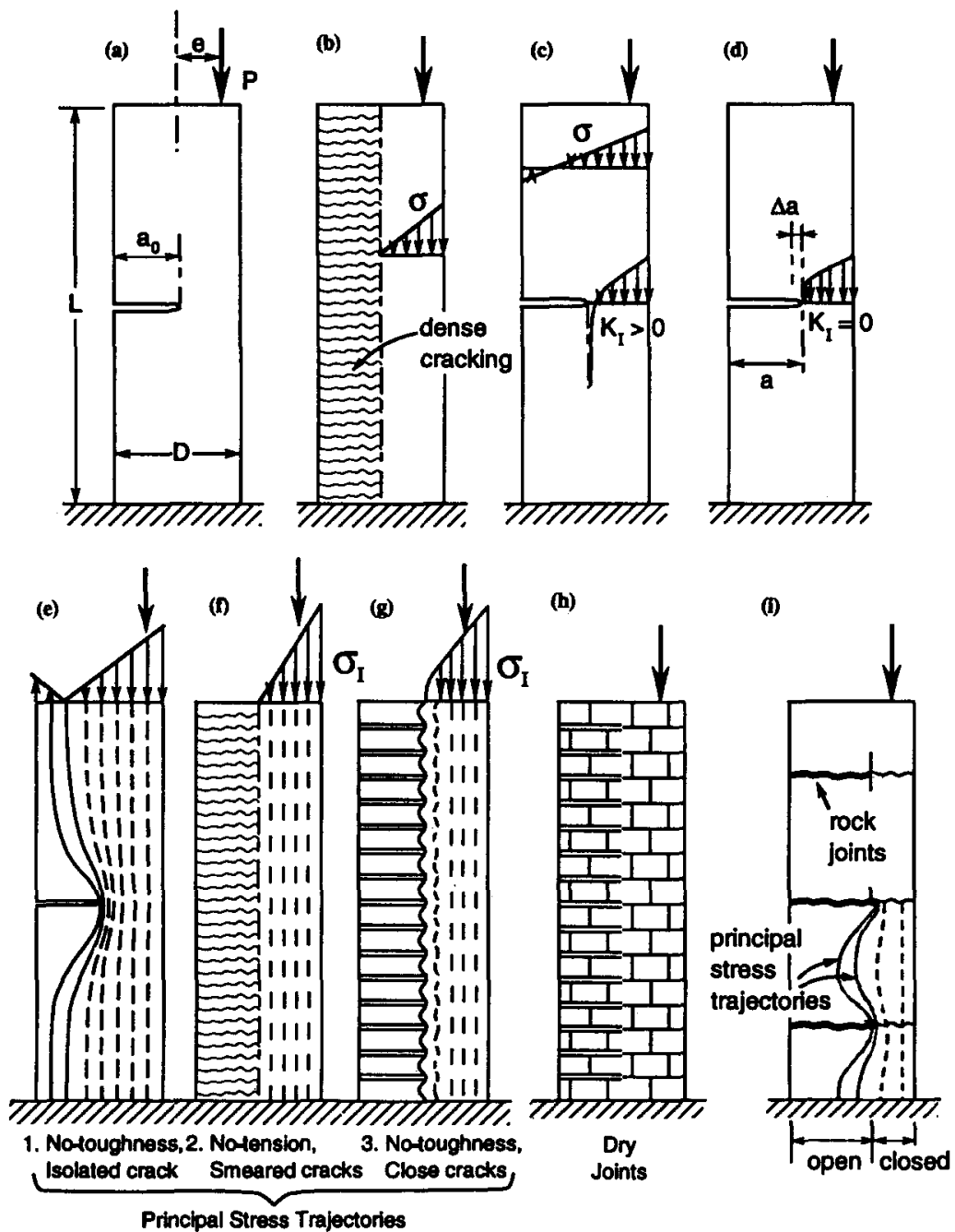

FIG. 1. (a) Rectangular Specimen Analyzed in Example 1; (b) Distributed Cracking Implied by No-Tension design; (c,d) Stress Distributions according to Fracture Mechanics with $K_{1}>0$ and $K_{1}=0$; (e) Approximate Trajectories of Minimum Principal Stresses for $K,>0$; (f-h) Masonry with Dry Joints; (I) Specimen of Jolnted Rock 
(or maximum) load $P_{u}$ is in practice assumed to occur when the maximum magnitude of the minimum (compressive) principal stress becomes equal to the allowable stress $\sigma_{\text {all }}=\mu f_{c}^{\prime}$, where $f_{c}^{\prime}$ is a safe, low estimate of the "compression strength" of the material and $1 / \mu$ is the safety factor, for which a rather high value is normally assumed.

The ultimate (maximum) load $P_{u}$ according to the elastoplastic design, based on a zero tensile yield strength and allowable compression stress, is the resultant of a triangular stress distribution [Fig. 1(b)]. So, $P_{u}=\mu f_{c}^{\prime} D / 4$. Obviously, this is a possible solution, satisfying all the conditions of equilibrium and compatibility. But this must also be the only possible no-tension solution, because the no-tension material is the special limiting case of an elastoplastic material, for which the solutions of boundary value problems (without nonlinear geometric effects) are known to be unique.

Second, consider the material to have a finite tensile strength, $f_{t}^{\prime}>0$. In that case, the left half of the specimen will not suffer continuously distributed cracking as shown in Fig. 1(a). Rather, a sharp crack will be present, as is often seen in dams. So fracture mechanics must be used to solve the problem. The solution can be obtained as the superposition of: (1) the solution for centric load $P$; and (2) the solution for moment $M=P e$ applied on the top of the specimen. These two solutions are available in handbooks (Tada et al. 1985; Murakami 1987 ), according to which the mode $I$ stress intensity factor at the crack tip is

$$
K_{l}=\sqrt{\frac{\pi \alpha}{D}}\left[\frac{6 M}{D} F_{M}(\alpha)+N F_{N}(\alpha)\right], \quad \alpha=\frac{a}{D}
$$

where, for $\alpha \leq 0.6$

$$
\begin{aligned}
& F_{M}(\alpha)=1.122-1.40 \alpha+7.33 \alpha^{2}-13.08 \alpha^{3} \\
& \quad+14.0 \alpha^{4}(\text { error } \leq 0.2 \%) \\
& F_{N}(\alpha)=1.122-0.231 \alpha+10.55 \alpha^{2}-21.72 \alpha^{3} \\
& \left.\quad+30.382 \alpha^{4} \text { (error } \leq 0.5 \%\right)
\end{aligned}
$$

and, for all $\alpha$

$$
\begin{aligned}
& F_{M}(\alpha)=\sqrt{\frac{2}{\pi \alpha} \tan \frac{\pi \alpha}{2}}\left(\cos \frac{\pi \alpha}{2}\right) \\
& \cdot\left[0.923+0.199\left(1-\sin \frac{\pi \alpha}{2}\right)^{4}\right] \text { error } \leq 0.5 \% \\
& F_{N}(\alpha)=\sqrt{\frac{2}{\pi \alpha} \tan \frac{\pi \alpha}{2}}\left(\cos \frac{\pi \alpha}{2}\right)^{-1}[0.752+2.02 \alpha \\
& \left.+0.37\left(1-\sin \frac{\pi \alpha}{2}\right)^{3}\right] \text { error } \leq 0.5 \%
\end{aligned}
$$

where $N=-P=$ normal force in the cross sections. The foregoing expressions apply for an infinitely long specimen $(H / L \rightarrow \infty)$, but they are very good approximations even for finite $H$, provided we assume $H / L \geq 4$. Note that $K_{I}$ characterizes the energy release rate $\mathscr{G}$ because $\mathscr{G}=K_{I}^{2}\left(1-v^{2}\right) /$ $E$, where $E=$ Young's modulus and $\nu=$ Poisson's ratio.

Substituting $N=-P$ and $M=P e$ with $P=\mu f_{c}^{\prime} D / 4$ (the load capacity for the no-tension design), we now evaluate $K_{I}$ for the case $\alpha=a / D=0.5$ [Fig. 1(a)]. The result is

$$
K_{I}=0.0505 f_{c}^{\prime} \sqrt{D}>0
$$

Now, an important point is that the stress intensity factor $K_{I}$ is positive. This means that, according to LEFM, the axial normal stresses $\sigma_{y}$ in the ligament of the cracked cross section tend to $+\infty$ as the crack tip is approached [Fig. 1(c)]. In practice, of course, $\sigma_{y}$ cannot exceed the local tensile strength of the material. But the positiveness of $K_{I}$ has two implications:

1. Tensile stresses exist within a certain portion of the horizontal cross section near the crack tip (in reality, of course, these stresses are not infinite, but the resultant of the actual tensile stresses is about the same as the resultant of the LEFM tensile stresses at the singularity). Consequently, for the given load $P$ with eccentricity $e=D / 3$ and the given crack length $a=D / 2$, the design is actually not a no-tension design if the finiteness of the material tensile strength is taken into consideration.

2. Growth of the crack would cause a release of energy from the structure. When a structure can release energy, it does so spontaneously (according to the second law of thermodynamics) and is unstable [Bažant and $\mathrm{Ce}$ dolin (1991), Chapters 12 and 13].

As an alternative to the no-tension design, in which the tensile strength of the material is also taken as zero but in a different sense, one may propose the concept of "no-toughness" design. In this design, the critical value $K_{I c}$ of the stress intensity factor, called the fracture toughness, is taken as zero (i.e., $K_{I c}=0$ ) and the stress at the crack tip cannot be unbounded [Fig. 1(d)]. If the fracture toughness is zero, the no-tension design that we obtained represents an unstable situation. The crack will propagate dynamically. The notoughness design is obtained for the crack length $a$ for which $K_{l}=0$.

From Fig. 2, we see that the curve of $K_{I}$ versus the relative crack length $\alpha=a / D$ has a zero point. So the no-toughness design exists, and Newton's iterative method yields the value

$$
\alpha=0.549
$$

Thus the stable crack is longer than that obtained by the notension design. If the crack extension from $\alpha=0.5$ to 0.549 occurs at a constant load-point displacement, the load must obviously decrease. Therefore, the load-deflection diagram for the no-toughness design lies below that for the no-tension design (Fig. 3).

In reality, the fracture toughness $K_{I c}$ is, of course, nonzero. Then the no-tension design may or might not yield a stable, safe state. But it is important to realize that, according to

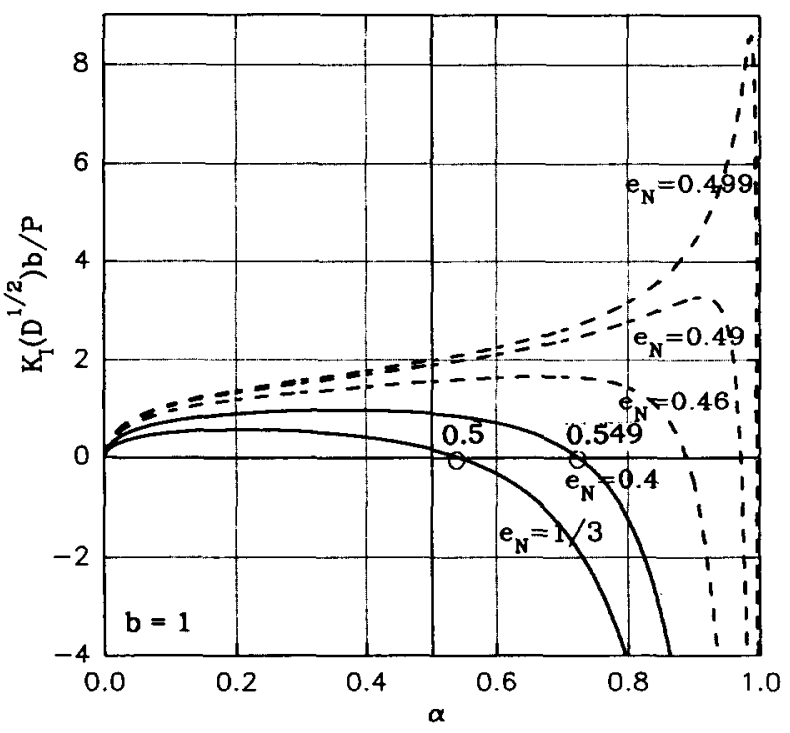

FIG. 2. Dependence of Stress Intensity Factor $K$, on Relative Crack Length $\alpha$ for Various Crack Lengths a and Corresponding Eccentricities $e_{N}$ of Compression Resultant 

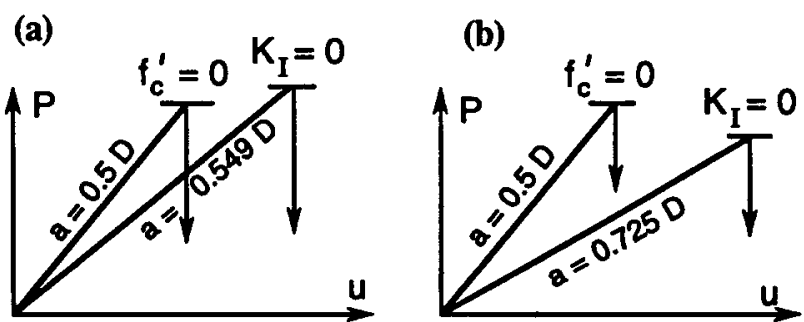

FIG. 3. Load-Deflection Curves for No-Tension and No-Toughness Designs

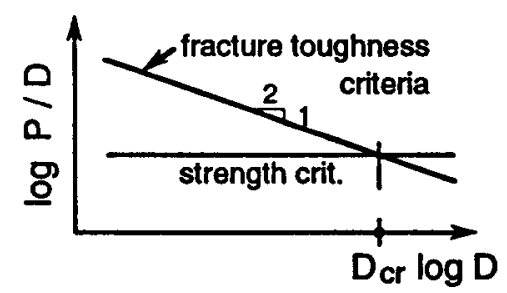

FIG. 4. Size Effect in Geometrically Similar Structures according to Strength Criteria and Linear Elastic Fracture Mechanics

(6), the no-tension design will yield an unstable state for any given value of $K_{I c}$ if the size $D$ exceeds a certain value, namely

$$
D>\left(\frac{K_{l c}}{0.0505 f_{c}^{\prime}}\right)^{2}
$$

This property is illustrated in Fig. 4 , in which $\log K_{I}$ is plotted versus $\log D$ for the plastic no-tension design and for LEFM. The plots are a horizontal line and a straight line of downward slope $-1 / 2$. Obviously, these two lines must intersect for a certain size $D$.

What do our results mean for rock? The no-tension concept is used in geotechnical engineering because rock joints can actually have zero tensile strength. However, the strength of the rock between the joints [Fig. $1(\mathrm{~g})$ ] is usually nonzero. So, the joints cannot be considered to be infinitely densely dis- tributed, as required for the applicability of the no-tension design. If our specimen were made of rock, the no-tension design would be justified only if the spacing of the joints (which are equivalent to cracks) were much smaller than the cross-section dimension $D$. This is normally true for masonry [Fig. 1(h)] but not for typical rock mechanics problems. The value of $a$ in our example represents the length of the open portion of the joint, and the rest of the joint transmits compressive stresses. At the end of the open portion we must have $K_{I}=0$ because the stresses cannot be infinite. Now, if the open portion of the joint has length $a=D / 2$, as predicted by the no-tension analysis of the joint, the resulting $K_{I}$ value for the end of the open portion is nonzero. The principal stress trajectories get concentrated near the front of the open portion of each no-tension joint, as approximately sketched in Fig. 1(i). Such an opening state of the joint is unstable. So the open portion of the joint will extend up to length $a=0.549 D$, for which $K_{1}=0$.

The situation is different when the joints of masonry have zero strength (dry joints) and are not far apart (relative to $D$ ), but densely distributed. In that case, the boundary of the compression zone of the cross section is indeed located at approximately $a=D / 2$. Tensile stresses between the joints (i.e., within the bricks) exist but are very small. The tensile principal stress trajectories are deflected by the fronts of the open portion of the joints, but only very little, as shown by the wavy line in Fig. $1(\mathrm{~g})$.

The axial stress distributions across the uncracked ligament, produced by load $P$ with eccentricity $e=D / 3$, have been obtained by the elastic finite-element analysis, both for $\alpha=0.549$ and $\alpha=0.5$ (Fig. 5). The stress profile for $\alpha=$ 0.5 in Fig. 5 illustrates the existence of tensile stresses near the crack tip.

For $\alpha=0.549$, we see no negative stresses. The stress profile terminates at the crack tip asymptotically as $\sigma \propto$ $\sqrt{x-a}$ (where coordinate $x$ is the distance from the crack mouth). This is the third term of the near-tip asymptotic series expansion (Broek 1986). The first term, proportional to $K_{l} /$ $\sqrt{x-a}$, vanishes because $K_{1}=0$. The second term, which

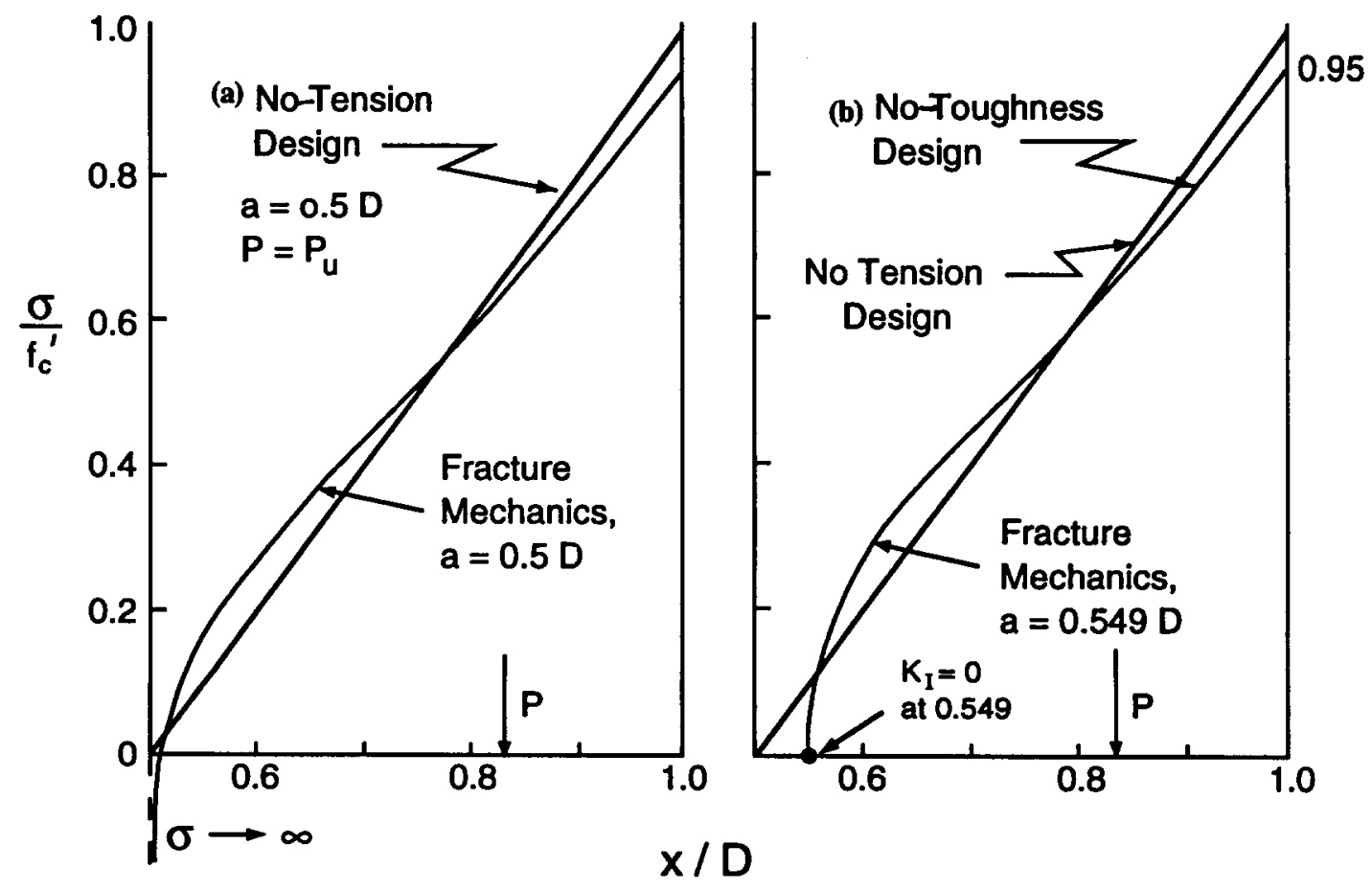

FiG. 5. Axial Stress Distributions along Ligament of Cracked Cross Section for Load $P$ with Eccentricity $e=D / 3(F i g .1)$ and Crack Lengths $a=0.5 D$ and $0.549 D$ 
is a constant, contributes only normal stresses parallel to the crack.

Fig. 5 reveals that, for the same $P$, the maximum compressive stress magnitude in the cross section is $95 \%$ of that for $\alpha=0.5$. So, according to the allowable compressive stress criterion, the load capacity for the no-toughness design is, in this example, $5 \%$ higher than for the no-tension design, even though the load-deflection diagram of the no-toughness design lies below that of the no-tension design (Fig. 3). So, in this example, the no-tension design yields a safe estimate of the load capacity.

This kind of conclusion, however, is not obtained in all situations, as shown in the following example. Besides, there is one questionable aspect: for the no-toughness design, the uncracked ligament of the cross section is smaller than for the no-tension design, yet the allowable compression stress criterion predicts the compression capacity of the ligament to be larger. This kind of conclusion is valid only under the hypothesis that the allowable stress is a realistic criterion for compression failure, which is not the case.

If the real compression failure mechanism, consisting of propagation of splitting compression cracks or shear damage bands, is taken into consideration, the compression failure, too, exhibits size effect. Thus the load capacity corresponding to compression failure must be expected to decrease with size $D$. In a manner similar to that in Fig. 4, for tensile failure, if a certain size is exceeded, the actual load capacity could become lower than the load capacity for no-tension analysis with allowable compression stress, which is known to exhibit no size effect.

The foregoing conclusion concerns only the overload by axial load $P$. If, subsequent to load $P$ causing the crack to extend up to length $a=0.549 D$, a horizontal shear force $P_{x}$ is superimposed as an additional load, the shear capacity of the specimen will be lower than that predicted by the notension design for the crack of length $a=0.5 D$. The reason is that the length of the uncracked ligament, $D-a=0.451 D$ [which is available to resist sliding due to shear loading; WES (1983)], is smaller than for the no-tension design, for which $D-a=0.5 D$. This conclusion is important for the earthquake loading of dams.

\section{EXAMPLE 2: REMOTE APPLIED FORCE AND CRACK FACE PRESSURE}

Let us now consider the same specimen with relative crack length $\alpha=0.5$, but with a different loading. In addition to the remotely applied axial force $P$, the crack faces (all the way to the crack tip) are loaded by uniform pressure $p$ due to water that penetrates the crack. Such loading occurs in cracked dams. Let us assume that $P$ and $p$ grow proportionally, setting $p=\rho P / D$ where $\rho$ is a given coefficient. For a typical dam profile, $\rho \approx 0.6$ at full reservoir. We will assume the value of $\rho$ to be precisely $\rho=0.6153$ (this number has been chosen simply because the finite-element results were already available for this value). The eccentricity of load $P$ (Fig. 6) is assumed to be precisely $e=0.1539 D$ [this is a value for which the loads applied on the upper half of the specimen above the cracked cross section in Fig. 6(a) are in balance with a triangular stress profile through the ligament, i.e., the uncracked part of the cross section].

First, consider no-tension plastic limit analysis. The fracture front cannot remain sharp, or else singular stresses tending to $+\infty$, which violate any strength limit, would develop and the no-tension analysis would thus predict the crack to run all the way across the ligament, for any positive load $P$, no matter how small. So, the crack front must widen. Thus, despite pressure $p$ on the crack faces, smeared (densely distributed) cracks must develop parallel to the crack faces [Fig. 6(c)]. This is possible if we consider the tensile strength to be zero and assume that water of pressure $p$ permeates also these parallel cracks.

The no-tension solution is easily found if the entire left half of the specimen is assumed to undergo smeared horizontal cracking. Then each horizontal cross section is in the same stress state and remains plane. Thus, the stress distribution across the right half of the cross section is triangular, as shown in Fig. 6(c) (at the limit state of no-tension design, the stress profile must have a jump from $-p$ to 0 at the cracking front). The vertical condition of equilibrium of the top half of the specimen yields $P-\rho P / 2=\mu f_{c}^{\prime} D / 4$, and so the ultimate load is

$$
P=P_{u}=0.3611 \mu f_{c}^{\prime} D
$$

Admittedly, the aforementioned process of spreading of pressurized cracks according to the zero tensile hypothesis involves conceptual difficulties. But example 2 is not irrelevant for current practice because no-tension finite-element calculations for dams with pressurized cracks have been carried out. In the discrete form (finite-element analysis), the conceptual difficulties we alluded to do not arise. But they cannot really be completely ignored because refinement of the element size to zero must yield the continuum solution.

Second, consider that the material tensile strength is not zero but finite, and the crack extension is governed by the LEFM fracture toughness criterion (which is equivalent to the energy release criterion). The finiteness of strength implies that the strip of width $a / 2$ (left half of the specimen) cannot be cracked in a continuous, smeared manner. When a crack forms, other cracks cannot form near it because the stress in the material on the sides of the crack is reduced. So, stress concentrations at the crack tip must arise and fracture mechanics must be used.

By virtue of the linearity of LEFM, the solution can be obtained by superposing the solutions of the two loading cases shown in Fig. 6(b): Case A, in which uniform pressure $p$ is applied on top of an uncracked specimen; and case $B$, in which uniform tension $p$ over the entire top and concentrated force $P$ of eccentricity $e$ are applied on the cracked specimen. The values of $K$, for these two cases are superimposed. For case $\mathrm{A}$, a state of homogeneous compression $\sigma=-p$ is obtained, and so $K_{I}=0$. Nonzero $K_{I}$ can be produced only in case $B$, which is again solved by (2)-(5). The axial force resultant and the bending moment to be substituted in these equations are $N=p D-P=(\rho-1) P$ (taken negative if compressive) and $M=P e$.

For a crack of length $a=0.5 D$ and the given loading with $P=P_{u}=0.6499 \mu f_{c}^{\prime} D,(2)-(5)$ again yield a positive $K_{r}$ value. So the crack that we found to be stable according to the no-tension design is again found to be unstable according to fracture mechanics and to propagate dynamically. (Even if the fracture toughness $K_{l c}$ were finite, the no-propagation criterion $K_{I} \leq K_{I c}$ would always be violated for a sufficiently large $D$, as before.)

The next question is whether a stable crack with $K_{I}=0$ exists for $0.5 D<a<D$. For the given load $P$ of eccentricity $e=0.1539 D$ and uniform crack face pressure $p=\rho P / D=$ $0.6153 P / D$ extending up to the tip, we can calculate from (2)(5) the curve of $K$, as a function of $\alpha$. To this end, we substitute the $M$ and $N$ values for the loading case $\mathrm{B}$, that is, moment $M=P e$ and centric force $N=p D-P=(\rho-$ 1) $P$ (where $N$ is taken as negative if compressive). This is equivalent to loading by axial force $N$ with eccentricity $e_{N}=$ $-M / N=e /(1-\rho)=0.1539 D /(1-0.6153)=0.4000 D$. The calculated curve of $K_{l}$ is shown in Fig. 2 for $e_{N} / D=0.4$. We see the curve has a zero point. This means that a stable 


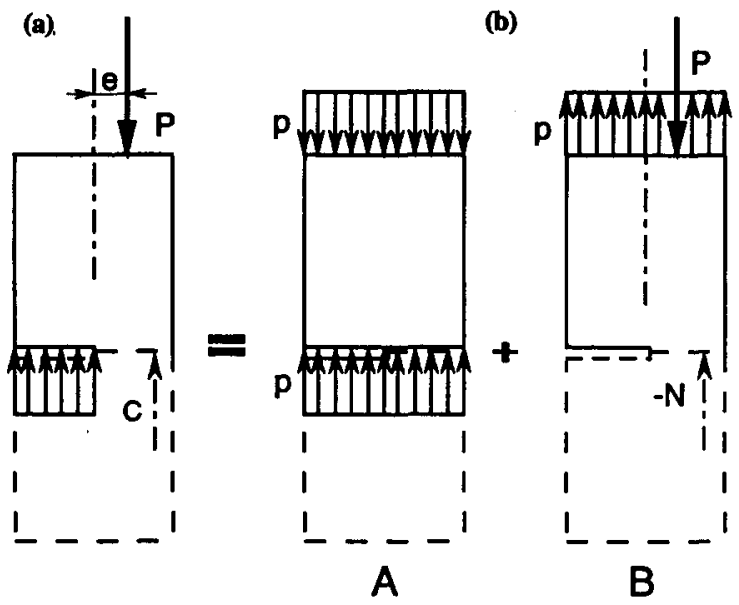

(c)

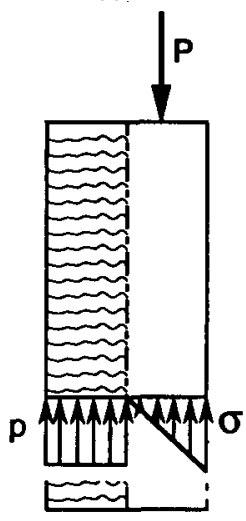

FIG. 6. (a) Rectangular Specimen with Crack Face Pressure p Analyzed in Example 2; (b) Equlvalent Superposition of Loading Cases A and B; (c) Stress Distribution in Case of Pressurized Densely Distributed Cracks

crack does exist for the given loading. Iterative solution of the root shows that $K_{I}=0$ occurs precisely for

$$
\alpha=0.725
$$

For this crack length and for zero toughness $\left(K_{I c}=0\right)$, the loads $P$ and $p$ that the specimen can carry are limited only by the capacity of the material to resist compression, which may be approximately characterized in terms of the allowable compression stress $\mu f_{c}^{\prime}$. The stress distribution across the uncracked ligament, which has been calculated by finite elements, is plotted in Fig. 7(b), in which the maximum compressive stress magnitude is $\sigma=-3.25 P / D$. Setting this equal to $\mu f_{c}^{\prime}$, we get

$$
P_{\max }=0.308 \mu f_{c}^{\prime} D
$$

This represents $85 \%$ of the ultimate load capacity $P_{u}$ predicted by the no-tension approach. So we have an example
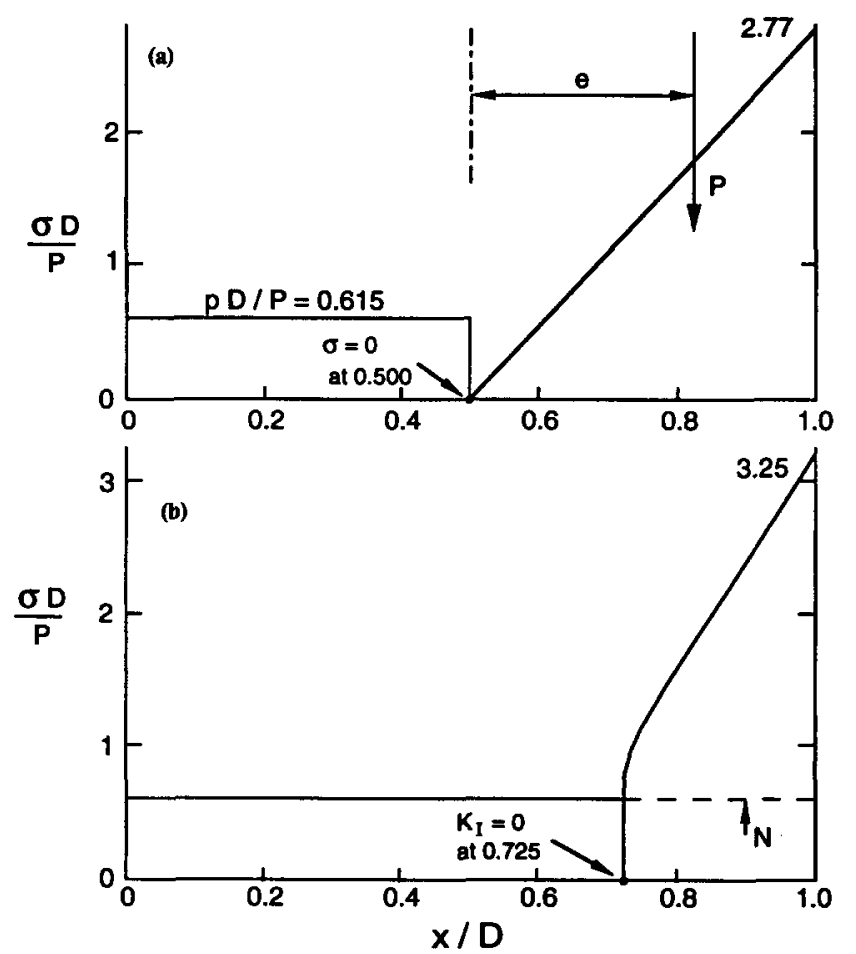

FIG. 7. Stress Distributions in Cracked Cross Section of Specimen with Crack Face Pressure (Example 2) for: (a) No-Tension Design; (b) No-Toughness Design that the no-tension design not only can yield an unstable crack, but also can significantly overpredict the load capacity of the structure.

Similar results can be obtained for other crack lengths. This is clear from the curves of $K_{I}$ versus $\alpha$ shown in Fig. 2 for various increasing values of $e_{N}$ corresponding to increasing values of $a$. As $a \rightarrow D$ (which corresponds to $e_{N} \rightarrow D / 2$ ), these curves approach at $x=D / 2$ a dipole, that is a jump from $+\infty$ to $-\infty$. The crack length $a<D / 2$ for which $K_{I}=$ 0 always exists and approaches $a \rightarrow D$. As $p D / P$ approaches 0.6922 , both $a_{0}$ for the no-tension design and $a$ for the notoughness design approach 1 . For $p D / P>1-(2 e / D)=$ 0.6922 , both the no-tension and no-toughness designs cease to exist.

It might seem strange that for $K_{I}=0$ the stress profile in Fig. 7(b) does not have a zero stress at the crack tip even though crack propagation is incipient. To understand that this must be so, observe two facts: (1) For a crack of length $a=$ $0.725 D(1+\delta)$ (where $\delta$ is arbitrarily small), the $K_{r}$ value would be positive, and thus LEFM would give an infinite tensile stress ahead of the tip [Fig. 8(a)], the physical meaning of which is that the energy release rate is nonzero; and (2) for a crack of length $a=0.725 D(1-\delta)$, the $K_{r}$-value would be negative, and thus LEFM would give an infinite compressive stress ahead of the tip [Fig. 8(c)], which would be impossible because it would imply an overlap of the crack faces. So, as $a$ grows, the LEFM stress must jump from $+\infty$ to $-\infty$ at $\alpha=0.725$. The difference from the critical stress profile for the no-tension limit design [Fig. 7(a)] illustrates Griffith's fundamental idea: the propagation of a sharp crack

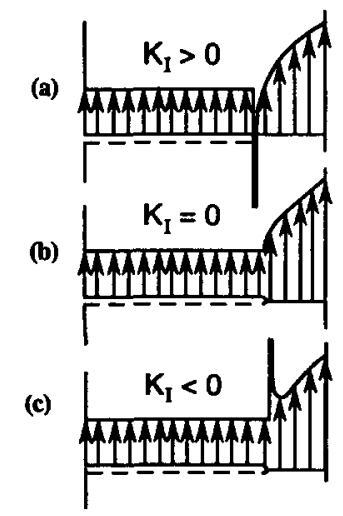

FIG. 8. Stress Distributions in Cracked Cross Section of Specimen with Crack Face Pressure (Example 2) for Various Values of Stress Intensity Factor $K$, 
must be decided by the critical energy release rate (or fracture toughness) rather than the material strength.

The loading with crack face pressure has the particular feature that, because of the difference in crack length, the ratio of the pressure resultant $p a$ to the axial load $P$ is not the same for the no-tension and no-toughness designs when the ratio of $p$ to $D$ is the same. Whether, for a loading without crack face pressure, the load capacity according to fracture mechanics could be less than the load capacity according to the no-tension design is not known at present. This question should be researched further.

\section{ANALYSIS OF PRESENT RESULTS}

Both examples demonstrate that the crack length obtained from the no-tension plastic design can be unstable if the finiteness of the material strength is taken into account. For zero fracture toughness, the crack is unstable in these examples for any size $D$, while for finite fracture toughness it becomes unstable only when a certain size $D$ is exceeded.

Example 2, as well as example 1 with horizontal load $P_{x}$ superimposed after crack formation, further demonstrates that the load capacity obtained by fracture mechanics can be less than that obtained by the no-tension design. This is true not only for the no-toughness design, but also, for a large enough structure, for a design with finite fracture toughness. As mentioned, this conclusion may be understood on observing that a structure of a nonzero tensile strength can store more energy than a structure of zero tensile strength, and can thus release energy during crack propagation at a higher rate.

Another simple, albeit partial, explanation is provided by the size effect. The plastic no-tension design exhibits no size effect, while failures controlled by fracture mechanics exhibit a size effect such that the nominal strength for similar failures of geometrically similar structures of different sizes decreases in inverse proportion to the structure size $D$, as illustrated in Fig. 4. From this it follows that if the cracks are geometrically similar, there must exist a structure size $D_{c r}$ for which the nominal strength of the structure becomes less than the strength obtained by the no-tension plasticity. However, this explanation does not apply if $K_{I}=0$ and if the load capacity is assumed to be controlled by the allowable compressive stress, for which there is no size effect.

The zero tensile strength assumption is nevertheless correct if the cracks are known to be densely distributed. In other words, the assumption is correct if the cracks do not localize. Aside from the case of dry joint masonry mentioned, this is the case for bending cracks in a reinforced-concrete beam, provided the reinforcement ratio exceeds a certain minimum value [Bažant and Cedolin (1991), Chapter 12].

The problem with the no-tension design may be intuitively understood from the shape of the principal stress trajectories, which are sketched for the case of cracked specimens with finite and zero tensile strengths in Fig. 1(e,f). The trajectories of the tensile and compressive principal stresses are shown by the solid and dashed lines. The tensile stress field develops high stress concentrations at places where the adjacent principal stress trajectories approach each other. The solid curves in Fig. 1(e), representing the tensile principal stress trajectories, are deflected by the crack tip because they must pass around it. This forces the trajectories to become very close to each other in the vicinity of the crack tip. By contrast, in the case of no-tension design, which corresponds to the continuously distributed cracking in the left half of the specimen in Fig. 1(b), all the principal stress trajectories are parallel vertical lines. The tensile response at zero tensile strength is of a plastic nature. Plasticity blunts a crack, forcing the cracking front to be smooth.

The present results further reveal an amazing effect: an increase in the tensile strength of the material can cause a reduction of the load capacity of the structure, with all other geometric and material characteristics being the same. In plasticity, this effect is impossible.

The basic conclusion from the present examples, as well as some recent finite-element studies (to be reviewed), is that the no-tension limit design of concrete or rock structures cannot guarantee that the actual safety factor will have the value specified by the building code. The no-tension design will nevertheless remain a useful tool, because of its simplicity. The classical applications of this design approach to dams or tunnels have no doubt been safe, especially in view of the large values of the safety factors used in design.

The present study nevertheless demonstrates that if the design is not based on fracture mechanics, it should at least be checked by fracture mechanics. Designing on the basis of fracture mechanics would make it possible to achieve more uniform safety margins and, thus, either permit a lowering of the safety factors or a further decrease in the already extremely small probabilities of failure.

\section{ANALYSIS OF PREVIOUS FINITE-ELEMENT RESULTS}

Most of the present observations have in essence already been made in the studies by Bažant (1990) and Gioia et al. (1992), although not on the basis of such simple examples. The Gioia et al. study involved finite-element calculations, which will be reviewed briefly, for the sake of completeness of the arguments.

A cracked specimen of finite length $L$ was considered subjected to a combination of axial load $P$ and bending moment $M$, which resembles the loading of a dam (Fig. 9). Using fracture mechanics solutions from handbooks and assuming that $K_{I}=1$, Bažant (1990) calculated the eccentricity $e$ of the compression resultant in the uncracked ligament of the cracked cross section. Various values of the relative crack length $a / D$ were considered. The distance of the compression resultant from the right side of the specimen is $(D / 2)-e$, while according to the plastic no-tension design, for which the stress distribution in the ligament is triangular, this distance is $(D-a) / 3$. Thus, the ratio

$$
\rho=\frac{(D / 2)-e}{(D-a) / 3}
$$

characterizes the change of the location of the compression resultant compared to the plastic no-tension design. In the case of a dam, the closer the compression resultant toward the end of the ligament, the lower the safety of the structure.

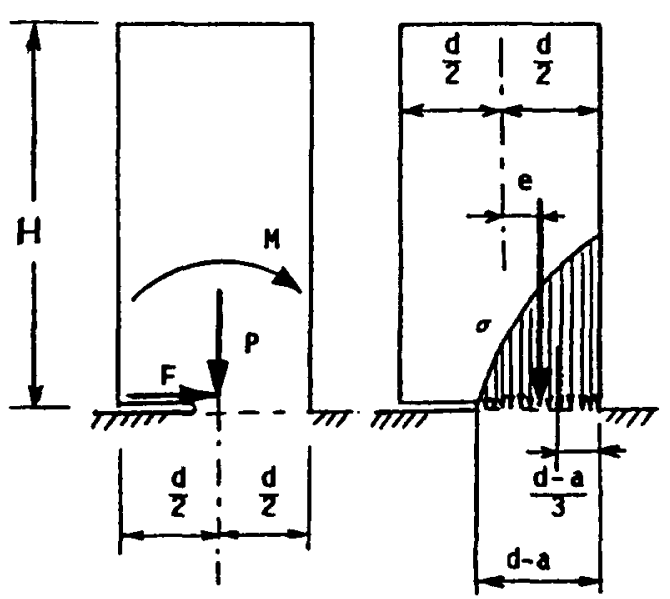

FIG. 9. Example Analyzed in Bažant (1990) and Location of Compression Resultant and Stress Distribution for $K_{1}=0$ 


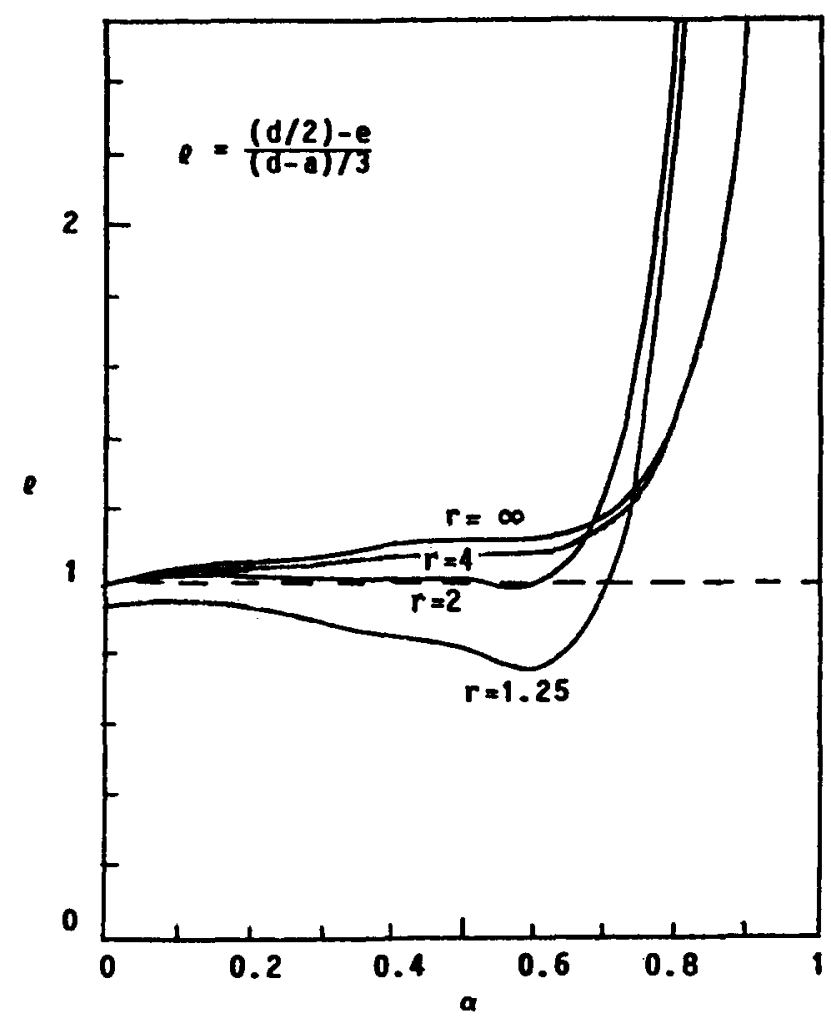

FIG. 10. Ratio $\rho$ of Distances of Compression Resultant from End of Llgament Calculated in Bažant (1990) for No-Toughness and NoTension Designs (Values $\rho<1$ Indicate Cases where No-Tension Design Gives Unsafe Estimate of Resultant Location)

Thus, the values $\rho \geq 1$ indicate the cases for which the plastic no-tension design yields the safe location of the compression resultant, and the values $\rho<1$ indicate the cases for which the plastic no-tension design yields an unsafe location. The values of the ratio $\rho$ as a function of the relative crack length are plotted in Fig. 10 for various values of the ratio $r-H /$ $D$, where $H=$ height of the specimen [for a table of the values of $\rho$, see Bažant (1990)]. There are many cases in which $\rho<1$.

A finite-element study of the safety of the no-tension design of dams was undertaken by Gioia et al. (1992). The geometry of the cross section of the Koyna dam (Saouma et al. 1990), which was stricken by an earthquake in 1967, was considered [Fig. 11(c) shows the finite-element mesh and the shape of the critical crack for the loading considered]. Finite-element solutions according to no-tension plasticity and according to fracture mechanics were compared [Fig. 11(c,d)]. The yield surface for no-tension plasticity [Fig. 11(a)] was a special case of Ottosen's (1977) yield surface for the tensile yield strength approaching zero. Because the origin of the stress space must lie inside the yield surface, the calculations have actually been run for a very small but nonzero value of the tensile yield strength of concrete, approximately 10 times smaller than a realistic value. Similarly, the no-toughness design was approximated in the finite-element calculations by taking the $K_{I C}$-value to be approximately 10 times smaller than the realistic value. The crack length obtained by fracture mechanics is, in this problem, very insensitive to the $K_{I c}$-value because $K_{I}$ represents a small difference of two large values $-K_{I}$ due to water pressure minus $K_{I}$ due to gravity load.

Similar to the present two examples, the differences between no-tension limit design and fracture mechanics have been found to be the most pronounced for the case when water penetrates into the crack and applies pressure on the crack faces. Because plastic analysis cannot describe crack propagation, the dam has been assumed to be precracked and loaded by water pressure along the entire crack length.

In the calculations, some of whose results are plotted in Fig. 11(c,d), the height of the water overflow above the crest of the dam was considered as the load parameter. A downward curving crack, which was indicated by calculations to be the most dangerous crack, was considered. From the results in Fig. 11(c), it is seen that the diagram of the load parameter, taken as the overflow height, versus the horizontal displacement at the top of the dam lies lower for fracture mechanics than it does for no-tension plasticity. In other words, the resistance offered by the dam to the loading by water is
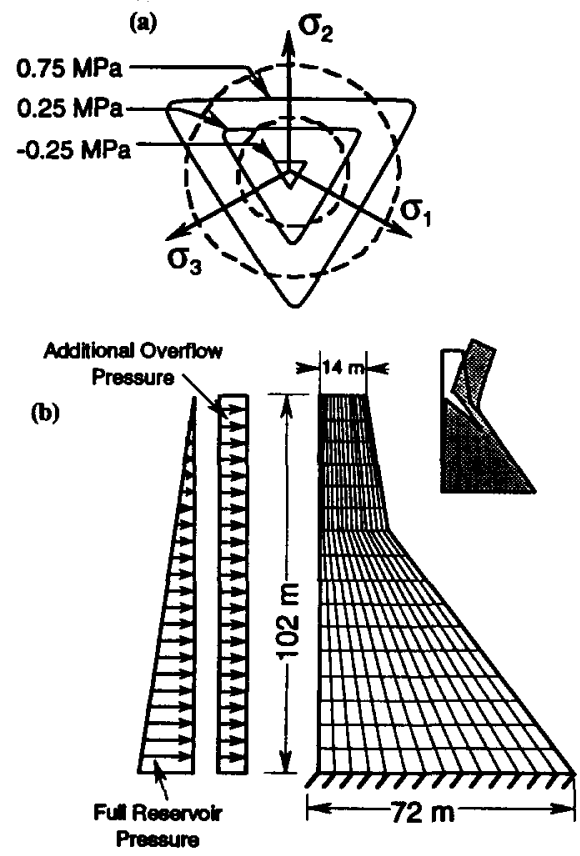
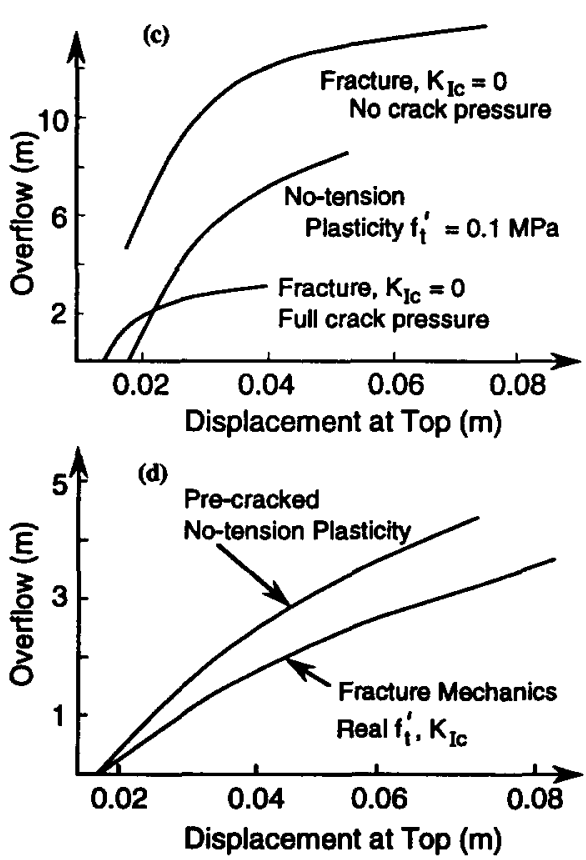

FIG. 11. Koyna Dam Analyzed in Gloia et al. (1992): (a) Yield Surfaces of Concrete; (b) Finite-Element Mesh and Failure Mode; (c) Comparison of Curves of Overflow Height versus Deflection for No-Tension Limit Analysis and No-Toughness Fracture Analysis; (d) Curves for Limit Analysis and Fracture Analysis with Realistic Values of Strength and Toughness 
lower according to the fracture mechanics solution, with a realistic value of the fracture toughness $K_{I c}$, than it is according to no-tension plasticity. Fig. 11(c,d) shows some typical calculated diagrams of load parameter versus displacement at the top of the dam for realistic values of the fracture toughness and the tensile yield strength $f_{i}^{\prime}$. It should be added that, for these finite-element calculations, the maximum of the load-deflection diagram could not be reached for realistic heights of overtopping of the dam. This is because the downward curvature of the critical crack tends to prevent the static load-deflection curve from flattening out. Thus, comparisons of the static load capacities were not possible for the geometry of the Koyna dam.

\section{CONCLUSIONS}

1. For a brittle (or quasi-brittle) elastic structure, the elastic-perfectly plastic analysis with a zero value of the tensile yield strength of the material is not guaranteed to be safe because it can happen that:

- The calculated length of cracks or cracking zones corresponds to an unstable state of crack propagation

- The uncracked ligament of the cross section, available for resisting horizontal sliding due to shear loads, is predicted much too large, compared to the fracture mechanics prediction

- The calculated load-deflection diagram lies lower than that predicted by fracture mechanics

- The load capacity for a combination of crack face pressure and loads remote from the crack front is predicted much too large, compared to the fracture mechanics prediction.

2. Due to the size effect, the preceding conclusions are true not only for zero fracture toughness (no-toughness design), but also for finite fracture toughness, provided the structure is large enough.

3. The no-tension limit design cannot always guarantee the safety factor of the structure to have the specified value. Fracture mechanics is required for that.

4. Increasing the tensile strength of the material can cause the load capacity of a brittle (or quasi-brittle) structure to decrease or even drop to zero.

5. The no-tension limit design would be correct if the tensile strength of the material were actually zero throughout the structure. This is true for dry masonry with sufficiently densely distributed joints, but not for concrete or for jointed rock masses.

6. The finiteness of the tensile strength of the material at points farther away from the cracks or rock joints (or construction joints) of negligible tensile strength causes the structure to store more strain energy. Thus, energy can be released during fracture propagation at a higher rate. This is one simple explanation of the present findings. Another simple explanation is the fact that a sharp crack tip causes stress concentrations, as manifested by crowding of the principal stress trajectories near the crack tip. When the material tensile strength is zero throughout the structure, the behavior is plastic in tension; this causes the cracking front to be smooth and thus prevents stress concentrations. Still another explanation (although only a partial one because it does not apply to failures controlled by allowable compressive stress) is the fact that failures controlled by strength criteria, even for zero tensile strength, exhibit no size effect whereas failures controlled by fracture mechanics do.

\section{ACKNOWLEDGMENTS}

Partial financial support under NSF Grant No. BCS-8818230 to Northwestern University is gratefully acknowledged. Thanks are due do YuanNeng Li, Research Assistant Professor, and Zhengzhi Li, Graduate Research Assistant, for their help in some of the computations.

\section{APPENDIX. REFERENCES}

Bažant, Z. P. (1984). "Size effect in blunt fracture: rock, concrete, metal." J. Engrg. Mech., ASCE, 110(4), 518-535.

Bažant, Z. P. (1990). "A critical appraisal of 'no-tension' dam design: a fracture mechanics viewpoint." Dam Engrg., 1(4), 237-247.

Bažant, Z. P. (1990). "Errata of 'a critical appraisal of "no-tension" dam design: a fracture mechanics viewpoint." Dam Engrg., 2(2).

Bažant, Z. P. (1994). "Recent advances in fracture mechanics, size effect and rate dependence of concrete: implication for dams." Proc., Int. Workshop on Dam Fracture and Damage, A. A. Balkema, Rotterdam, The Netherlands, 41-54.

Bažant, Z. P., and Cedolin, L. (1991). Stability of structures: elastic, inelastic, fracture and damage theories. Oxford University Press, New York, N.Y.

Bažant, Z. P., and Kazemi, M. T. (1990). "Size effect in fracture of ceramics and its use to determine fracture energy and effective process zone length." J. Am. Ceramic Soc., 73(7), 1841-1853.

Bažant, Z. P., He, S., Plesha, M. E., and Rowlands, R. E. (1991). "Rate and size effect in concrete fracture: implications for dams." Proc., Int. Conf. on Dam Fracture; Publ. 95-7491, V. Saouma, R. Dungar, and D. Morris, eds., Electric Power Res. Inst., Palo Alto, Calif, , 413-425

Bourdarot, E., Mazars, J., and Saouma, V. E. (eds.). (1994). “Dam fracture and damage." Proc. Int. Workshop on Dam Fracture and Damage, A. A. Balkema, Rotterdam, The Netherlands.

Broek, D. (1986). Elementary engineering fracture mechanics, 4th Ed., Martinus Nijhoff Publishers, Dordrecht, The Netherlands.

Brown, W. F., and Srawley, J. E. (1967). "Fracture toughness testing methods." Fracture toughness testing and its applications; STP 381, ASTM, Philadelphia, Pa.

Bureau of Reclamation (BuRec). (1987). Design of small dams, U.S. Dept. of the Interior, Denver, Colo.

Dungar, R., Saouma, V. E., and Wittmann, F. H. (eds.). (1990). Workshop notes: research workshop on "Application of fracture mechanics to dam engineering." Swiss Federal Inst. of Technol. (ETH), Zürich, Switzerland.

Gioia, G., Bažant, Z. P., and Pohl, B. (1992). "Is no-tension dam design always safe?" Dam Engrg., 3(1), 23-34.

Gioia, G., Bažant, Z. P., and Pohl, B. (1992). "Closure to "Is no-tension dam design always safe?', Dam Engrg., 4(1), 59.

Gross, B., and Srawley, J. E. (1964). "Stress intensity factors for a singleedge-notch tension specimen by boundary collocation of a stress function." Rep. NASA TN D-2395.

He, S., Plesha, M. E., Rowlands, R. E., and Bažant, Z. P. (1992). "Fracture energy tests of dam concrete with rate and size effects." Dam Engrg. , 3(2), 139-159.

Hodge, P. G. (1959). Plastic analysis of structures, McGraw-Hill Book Co., Inc., New York, N.Y.

Jansen, R. B. (1988). "Advanced dam engineering for design, construction and rehabilitation." Rep., U.S. Dept. of Interior, Denver, Colo.

Murakami, Y. (ed.) (1987). Stress intensity factors handbook; Vol. I, Pergamon Press, Oxford, England.

Ottosen, N. S. (1977). "A failure criterion for concrete." J. Engrg. Mech., ASCE, 103(4), 527-535.

Owen, D. R. J., and Hinton, E. (1980). Finite elements in plasticity, Pineridge Press, Swansea, Wales.

Saouma, V. E., Dungar, R., and Morris, D. (eds.). (1991). "Dam fracture." Proc., Int. Conf. on Dam Fracture; Publ. GS-7491, Electric Power Res. Inst., Palo Alto, Calif.

Saouma, V. E., Brühwiler, E., and Boggs, H. L. (1990). "A review of fracture mechanics applied to concrete dams." Dam Engrg., 1(1), 41-58.

Tada, H., Paris, P. C., and Irwin, J. K. (1985). The stress analysis of cracks handbook, 2nd Ed., Paris Productions, Inc., St. Louis, Mo.

Tardieu, B., and Londe, P. (1992). "Discussion of Bažant, Gioia and Pohl (1992)." Dam Engrg., 3(4), 301.

Waterways Experiment Station (WES). (1983). "Design of gravity dams on rock foundations: sliding stability assessment by limit equilibrium and selection of shear strength parameters." Tech. Rep. GL-83-13, U.S. Army Corps of Engrs., Vicksburg, Miss.

Zienkiewicz, O. C. (1971). The finite element method in engineering science, McGraw-Hill Book Co., Inc., London, England. 